International Journal of Pure and Applied Mathematics

Volume 96 No. $2 \quad 2014,189-196$

ISSN: 1311-8080 (printed version); ISSN: 1314-3395 (on-line version)

url: http://www.ijpam.eu

doi: http://dx.doi.org/10.12732/ijpam.v96i2.3

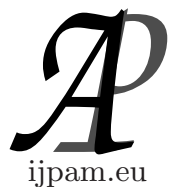

\title{
GENERALIZED DIFFERENCES AND
}

\section{ABEL'S TYPE BINOMIAL THEOREMS}

\author{
Ken Kuriyama $^{1}$, Shigeru Furuichi ${ }^{2} \S$ \\ ${ }^{1}$ Faculty of Education \\ Bukkyo University \\ Kyoto City, Kyoto, 603-8301, JAPAN \\ ${ }^{2}$ Department of Information Science \\ College of Humanities and Sciences \\ Nihon University \\ 3-25-40, Sakurajyousui, Setagaya-ku, Tokyo, 156-8550, JAPAN
}

Abstract: The first author proved Abel's type binomial theorems for the falling and/or rising factorial power in the previous paper [3]. The purpose of this paper is to give generalizations for these theorems by the use of the generalized forward and/or backward differences.

AMS Subject Classification: 05A10, 11B65

Key Words: generalized difference, generalized factorial power and Abel's type binomial theorem

\section{Introduction}

For an integer $n$, the falling factorial power $x^{\underline{n}}$ is defined as

$$
x^{\underline{n}}= \begin{cases}x(x-1)(x-2) \cdots(x-n+1) & (n=1,2,3, \cdots) \\ 1 & (n=0) \\ \frac{1}{(x+1)(x+2) \cdots(x-n)} & (n=-1,-2,-3, \cdots) .\end{cases}
$$

Similarly the rising factorial power $x^{\bar{n}}$ is defined as

Received: February 21, 2014

(C) 2014 Academic Publications, Ltd. url: www.acadpubl.eu

${ }^{\S}$ Correspondence author 


$$
x^{\bar{n}}= \begin{cases}x(x+1)(x+2) \cdots(x+n-1) & (n=1,2,3, \cdots) \\ 1 & (n=0) \\ \frac{1}{(x-1)(x-2) \cdots(x+n)} & (n=-1,-2,-3, \cdots) .\end{cases}
$$

The binomial theorems for the falling factorial power and the rising factorial power are known as $[2,3,5]$ :

$$
(x+y)^{\underline{n}}=\sum_{i=0}^{n}\left(\begin{array}{c}
n \\
i
\end{array}\right) x^{\underline{i}} y \underline{\underline{n-i}}, \quad \text { and } \quad(x+y)^{\bar{n}}=\sum_{i=0}^{n}\left(\begin{array}{c}
n \\
i
\end{array}\right) x^{\bar{i}} y^{\overline{n-i}} .
$$

In the previous paper [3], the first author of this paper proved the following Abel's type binomial theorems for the falling and rising factorial power:

$$
\begin{aligned}
& (x+y)^{\underline{n}}=\sum_{i=0}^{n}\left(\begin{array}{c}
n \\
i
\end{array}\right) x(x-1+i z)^{\frac{i-1}{n}}(y-i z) \frac{n-i}{n}, \\
& (x+y)^{\bar{n}}=\sum_{i=0}^{n}\left(\begin{array}{c}
n \\
i
\end{array}\right) x(x+1+i z)^{\overline{i-1}}(y-i z)^{\overline{n-i}} .
\end{aligned}
$$

These formulas (1) and (2) are generalizations for the Abel's generalized formula [1] given by

$$
(x+y)^{n}=\sum_{i=0}^{n}\left(\begin{array}{c}
n \\
i
\end{array}\right) x(x+i z)^{i-1}(y-i z)^{n-i} .
$$

At the same time, those formulas (1) and (2) are generalizations of the binomial theorems for falling (rising) factorial power.

In this paper, we prove the following theorem which is a further generalization of (1) and (2).

Theorem 1. Let $n$ be a natural number and $\alpha$ a complex number, then the following equalities hold.

$$
\begin{aligned}
& (x+y)^{\underline{n}, \alpha}=\sum_{i=0}^{n}\left(\begin{array}{c}
n \\
i
\end{array}\right) x(x-\alpha+i z)^{\underline{i-1}, \alpha}(y-i z) \underline{\underline{n-i, \alpha}}, \\
& (x+y)^{\bar{n}, \alpha}=\sum_{i=0}^{n}\left(\begin{array}{c}
n \\
i
\end{array}\right) x(x+\alpha+i z)^{\overline{i-1}, \alpha}(y-i z)^{\overline{n-i}, \alpha} .
\end{aligned}
$$

In the above theorem we set a generalized falling factorial power $x^{\underline{n}, \alpha}$ for any complex number $\alpha$ is defined as follows:

$$
x^{\underline{n}, \alpha}= \begin{cases}x(x-\alpha)(x-2 \alpha) \cdots(x-(n-1) \alpha) & (n=1,2,3, \cdots) \\ 1 & (n=0) \\ \frac{1}{(x+\alpha)(x+2 \alpha) \cdots(x-n \alpha)} & (n=-1,-2,-3, \cdots) .\end{cases}
$$


Similarly a generalized rising factorial power $x^{\bar{n}, \alpha}$ is defined as follows:

$$
x^{\bar{n}, \alpha}= \begin{cases}x(x+\alpha)(x+2 \alpha) \cdots(x+(n-1) \alpha) & (n=1,2,3, \cdots) \\ 1 & (n=0) \\ \frac{1}{(x-\alpha)(x-2 \alpha) \cdots(x+n \alpha)} & (n=-1,-2,-3, \cdots) .\end{cases}
$$

For the convenience of the reader's help to understand Theorem 1, we give the following example.

Example 1. We consider the case of $n=4$ in Theorem 1 . The equality (3) is calculated by

$$
\begin{aligned}
&(x+y)(x+y-\alpha)(x+y-2 \alpha)(x+y-3 \alpha) \\
&= y(y-\alpha)(y-2 \alpha)(y-3 \alpha)+4 x(y-z)(y-z-\alpha)(y-z-2 \alpha) \\
&+6 x(x+2 z-\alpha)(y-2 z)(y-2 z-\alpha)+4 x(x+3 z-\alpha)(x+3 z-2 \alpha)(y-3 z) \\
&+x(x+4 z-\alpha)(x+4 z-2 \alpha)(x+4 z-3 \alpha) .
\end{aligned}
$$

The equality (4) is also calculated by

$$
\begin{aligned}
&(x+y)(x+y+\alpha)(x+y+2 \alpha)(x+y+3 \alpha) \\
&= y(y+\alpha)(y+2 \alpha)(y+3 \alpha)+4 x(y-z)(y-z+\alpha)(y-z+2 \alpha) \\
&+6 x(x+2 z+\alpha)(y-2 z)(y-2 z+\alpha)+4 x(x+3 z+\alpha)(x+3 z+2 \alpha)(y-3 z) \\
&+x(x+4 z+\alpha)(x+4 z+2 \alpha)(x+4 z+3 \alpha) .
\end{aligned}
$$

\section{Generalized Differences and their Properties}

Let $\mathbb{C}$ be the field of complex numbers and $\mathrm{C}[x]$ the polynomial algebra over $\mathbb{C}$. We consider $\mathrm{C}[x]$ a linear space over $\mathbb{C}$. For $a \in \mathbb{C}$, shift operator $E_{a}$ is defined as follows: $E_{a}: \mathrm{C}[x] \ni f(x) \mapsto f(x+a) \in \mathrm{C}[x]$.

Definition 1. A linear operator $Q: \mathrm{C}[x] \rightarrow \mathrm{C}[x]$ is called a delta operator, if the following conditions are satisfied:

(1) if $f \in \mathrm{C}[x]$ is degree of $n$, then $Q f$ is degree of $n-1$,

(2) $Q$ commutes with all shift operators, that is, $Q E_{a}=E_{a} Q$ for all $a \in \mathbb{C}$.

We define a generalized forward difference $\Delta^{+, \alpha}$ and a generalized backward difference $\Delta^{-, \alpha}$ for any complex number $\alpha$ as follows:

$$
\begin{array}{ll}
\Delta^{+, \alpha} f(x)=f(x+\alpha)-f(x) & \text { for } \quad f \in \mathrm{C}[x], \\
\Delta^{-, \alpha} f(x)=f(x)-f(x-\alpha) & \text { for } \quad f \in \mathrm{C}[x] .
\end{array}
$$


In particular, if $\alpha=1$ then the generalized forward (backward) difference is a standard forward (backward) difference. That is, $\Delta^{+, 1} f(x)=f(x+1)-f(x)$ and $\Delta^{-, 1} f(x)=f(x)-f(x-1)$. Generalized forward differences and generalized backward differences are delta operators.

Proposition 1. We have the following equalities.

(1) For a generalized forward difference $\Delta^{+, \alpha}$ and $a \in \mathbb{C}$, we have

$$
\Delta^{+, \alpha}(x+a)^{\underline{n}, \alpha}=n \alpha(x+a)^{\frac{n-1}{2}, \alpha}, \quad(n=1,2, \cdots) .
$$

In particular, $\Delta^{+, \alpha} x^{\underline{n}, \alpha}=n \alpha x \underline{n-1}, \alpha,(n=1,2, \cdots)$.

(2) For a generalized backward difference $\Delta^{-, \alpha}$ and $a \in \mathbb{C}$, we have

$$
\Delta^{-, \alpha}(x+a)^{\bar{n}, \alpha}=n \alpha(x+a)^{\overline{n-1}, \alpha}, \quad(n=1,2, \cdots) .
$$

In particular, $\Delta^{-, \alpha} x^{\bar{n}, \alpha}=n \alpha x^{\overline{n-1}, \alpha},(n=1,2, \cdots)$.

Proof. (1) By simple calculations, firstly we can prove the equality $\Delta^{+, \alpha} x^{\underline{n}, \alpha}=$ $n \alpha x \stackrel{n-1, \alpha}{ }$. Since $Q \equiv \Delta^{+, \alpha}$ commutes with any shift operator $E_{a}$, that is, $Q E_{a}=E_{a} Q$, we then have

$$
\begin{aligned}
Q(x+a)^{\underline{n}, \alpha} & =Q E_{a} x^{\underline{n}, \alpha}=E_{a} Q x^{\underline{n}, \alpha} \\
& =E_{a}(n \alpha x \underline{\underline{n-1}, \alpha})=n \alpha E_{a} x \underline{\underline{n-1}, \alpha}=n \alpha(x+a) \underline{\underline{n-1}, \alpha} .
\end{aligned}
$$

Similarly we can prove (2).

\section{Proposition 2. (Product rule of generalized differences)}

(1) The equality $\Delta^{+, \alpha}\{f(x) g(x)\}=\left(\Delta^{+, \alpha} f(x)\right) g(x)+f(x+\alpha)\left(\Delta^{+, \alpha} g(x)\right)$ holds. In particular, $\Delta^{+, \alpha}\{x f(x)\}=\alpha f(x)+(x+\alpha)\left(\Delta^{+, \alpha} f(x)\right)$.

(2) The equality $\Delta^{-, \alpha}\{f(x) g(x)\}=\left(\Delta^{-, \alpha} f(x)\right) g(x)+f(x-\alpha)\left(\Delta^{-, \alpha} g(x)\right)$ holds. In particular, $\Delta^{-, \alpha}\{x f(x)\}=\alpha f(x)+(x-\alpha)\left(\Delta^{-, \alpha} f(x)\right)$.

Proof. (1) By simple calculations, we get

$$
\begin{aligned}
\Delta^{+, \alpha}\{f(x) g(x)\} & =f(x+\alpha) g(x+\alpha)-f(x) g(x) \\
& =(f(x+\alpha)-f(x)) g(x)-f(x+\alpha) g(x)+f(x+\alpha) g(x+\alpha) \\
& =(f(x+\alpha)-f(x)) g(x)+f(x+\alpha)(g(x+\alpha)-g(x)) \\
& =\left(\Delta^{+} f(x)\right) g(x)+f(x+1)\left(\Delta^{+} g(x)\right) .
\end{aligned}
$$


In the above equality, let $f(x)=x, g(x)=f(x)$. Since $\Delta^{+, \alpha} x=\alpha$, we have $\Delta^{+, \alpha}\{x f(x)\}=\left(\Delta^{+, \alpha} x\right) f(x)+(x+\alpha)\left(\Delta^{+, \alpha} f(x)\right)=\alpha f(x)+(x+\alpha)\left(\Delta^{+, \alpha} f(x)\right)$. Similarly we can prove (2).

\section{Proof of Theorem 1}

In this section, we prove Theorem 1 . For this purpose, we prepare the following lemmas. Let polynomials $A_{k}(x, z),(k=0,1,2, \cdots)$ in $x, z$ be as follows: $A_{k}(x, z)=x(x-\alpha+k z) \stackrel{k-1}{,}, \alpha$, with the convention $A_{0}(0, z) \equiv 1$. That is,

$$
\begin{aligned}
& A_{0}(x, z)=1, A_{1}(x, z)=x, \\
& A_{2}(x, z)=x(x-\alpha+2 z)^{\frac{1}{1} \alpha}=x(x-\alpha+2 z), \\
& A_{3}(x, z)=x(x-\alpha+3 z)^{2}, \alpha=x(x-\alpha+3 z)(x-2 \alpha+3 z),
\end{aligned}
$$

Lemma 1. Let polynomials $A_{k}(x, z)=x(x-\alpha+k z) \underline{k-1}, \alpha$ in $x, z,(k=$ $0,1,2, \cdots)$. If $f(x) \in \mathrm{C}[x]$ is a polynomial in $\mathrm{x}$ of degree $n$, then

$$
\begin{aligned}
f(x) & =\sum_{k=0}^{n} \frac{1}{\alpha^{k}} \frac{1}{k !}\left(\Delta_{x}^{+, \alpha}\right)^{k} f(-k z) A_{k}(x, z) \\
& =\sum_{k=0}^{n} \frac{1}{\alpha^{k}} \frac{1}{k !}\left(\Delta_{x}^{+, \alpha}\right)^{k} f(-k z) x(x-\alpha+k z) \frac{k-1, \alpha}{,},
\end{aligned}
$$

where $\Delta_{x}^{+, \alpha}$ is a generalized forward difference with respect to $x$, given by $\Delta_{x}^{+, \alpha} f(x) \equiv f(x+\alpha)-f(x)$.

Proof. We prove this lemma along to the following four steps.

(i). First we should remark that the polynomial $A_{k}(x, z)$ is degree of $k$ with respect to $x$ and $A_{k}(0, z)=0,(k=1,2, \cdots)$.

(ii). Let $\Delta_{x}^{+, \alpha}$ be a generalized forward difference with respect to $x$. We prove the following equality.

$$
\Delta_{x}^{+, \alpha} A_{k}(x, z)= \begin{cases}k \alpha A_{k-1}(x+z, z) & (k=1,2, \cdots), \\ 0 & (k=0) .\end{cases}
$$


From Proposition 1 and 2 (product rule of generalized differences),

$$
\begin{aligned}
& \Delta_{x}^{+, \alpha} A_{k}(x, z)=\Delta_{x}^{+, \alpha}\{x(x-\alpha+k z) \stackrel{k-1}{, \alpha}\} \\
& =\alpha(x-\alpha+k z) \frac{k-1}{2} \alpha+(x+\alpha) \Delta_{x}^{+, \alpha}\{(x-\alpha+k z) \stackrel{k-1, \alpha}{ }\} \\
& =\alpha(x-\alpha+k z)^{\frac{k-1}{2}} \alpha+(x+\alpha) \alpha(k-1)(x-\alpha+k z) \underline{k-2}, \alpha \\
& =\alpha(x-\alpha+k z) \stackrel{k-2}{2} \alpha(x-\alpha+k z-(k-2) \alpha)+(k-1)(x+\alpha)\} \\
& =k \alpha(x+z)(x-\alpha+k z) \underline{k-2}, \alpha .
\end{aligned}
$$

Hence $\Delta_{x}^{+, \alpha} A_{k}(x, z)=k \alpha(x+z)(x-\alpha+k z) \underline{k-2, \alpha}$.

Since $A_{k-1}(x, z)=x(x-\alpha+(k-1) z) \underline{k-2}, \alpha$,

$$
\begin{aligned}
A_{k-1}(x+z, z) & =(x+z)(x+z-\alpha+(k-1) z) \underline{k-2}, \alpha \\
& =(x+z)(x-\alpha+k z) \underline{k-2}, \alpha
\end{aligned}
$$

Therefore $\Delta_{x}^{+, \alpha} A_{k}(x, z)=k \alpha(x+z)(x-\alpha+k z) \underline{k-2, \alpha}=k \alpha A_{k-1}(x+z, z)$.

(iii). Using the above (ii) repeatedly, we get for $m=1,2, \cdots, k$

$$
\left(\Delta_{x}^{+}\right)^{m} A_{k}(x, z)=k(k-1) \cdots(k-m+1) \alpha^{m} A_{k-m}(x+m z, z) .
$$

In fact, we assume the above equality holds for some $m$. Then we have

$$
\begin{aligned}
\left(\Delta_{x}^{+, \alpha}\right)^{m+1} A_{k}(x, z) & =\Delta_{x}^{+, \alpha}\left\{\left(\Delta_{x}^{+, \alpha}\right)^{m} A_{k}(x, z)\right\} \\
& =\Delta_{x}^{+, \alpha}\left\{k(k-1) \cdots(k-(m-1)) \alpha^{m} A_{k-m}(x+m z, z)\right\} \\
& =k(k-1) \cdots(k-(m-1)) \alpha^{m} \Delta_{x}^{+, \alpha} A_{k-m}(x+m z, z) \\
& =k(k-1) \cdots(k-m) \alpha^{m+1} A_{k-(m+1)}(x+(m+1) z, z) .
\end{aligned}
$$

Thus we find that the equality (6) holds by induction on $m$.

(iv). Let $f(x)$ be a polynomial $f(x) \in \mathrm{C}[x]$ of degree $n$. Since $A_{k}(x, z)$ is a polynomial of degree $k$ with respect to $x$ and $f(x)$ is a polynomial of degree $n$, we can set

$$
f(x)=\sum_{k=0}^{n} a_{k}(z) A_{k}(x, z)
$$

Substituting $x=0$ in the equality (7), from the fact that $A_{0}(0, z)=1, A_{k}(0, z)=$ $0,(k=1,2, \cdots)$, we get $a_{0}(z)=f(0)$. Operating $\Delta_{x}^{+, \alpha}$ to the both sides of the equality (7),

$$
\begin{aligned}
\Delta_{x}^{+, \alpha} f(x) & =\Delta_{x}^{+, \alpha}\left\{\sum_{k=0}^{n} a_{k}(z) A_{k}(x, z)\right\} \\
& =\sum_{k=0}^{n} a_{k}(z) \Delta_{x}^{+, \alpha} A_{k}(x, z)=\sum_{k=1}^{n} a_{k}(z) k \alpha A_{k-1}(x+z, z)
\end{aligned}
$$


Substituting $x=-z$ in the above equality,

$$
\Delta_{x}^{+, \alpha} f(-z)=\sum_{k=1}^{n} a_{k}(z) k \alpha A_{k-1}(0, z)=\alpha a_{1}(z)
$$

Similarly operating $\left(\Delta_{x}^{+, \alpha}\right)^{m}$ to the both sides of the equality (7), we obtain with the repeated computations,

$$
\left(\Delta_{x}^{+, \alpha}\right)^{m} f(x)=\sum_{k=m}^{n} a_{k}(z) k(k-1) \cdots(k-(m-1)) \alpha^{m} A_{k-m}(x+m z, z) .
$$

Substituting $x=-m z$ in the above equality,

$$
\begin{aligned}
\left(\Delta_{x}^{+, \alpha}\right)^{m} f(-m z) & =\sum_{k=m}^{n} a_{k}(z) k(k-1) \cdots(k-(m-1)) \alpha^{m} A_{k-m}(0, z) \\
& =m(m-1) \cdots 2 \cdot 1 \cdot \alpha^{m} a_{m}(z)
\end{aligned}
$$

Therefore we have $a_{m}(z)=\frac{1}{\alpha^{m}} \frac{1}{m !}\left(\Delta_{x}^{+, \alpha}\right)^{m} f(-m z)$ for $m=1,2, \cdots, n$.

We also have the following lemma which can be proven by the similar way to the proof of Lemma 1 with the generalized backward difference $\Delta_{x}^{-, \alpha}$.

Lemma 2. Let polynomials $B_{k}(x, z)=x(x+\alpha+k z)^{\overline{k-1}, \alpha}$ in $x, z,(k=$ $0,1,2, \cdots)$. If $g(x) \in \mathrm{C}[x]$ is a polynomial in $\mathrm{x}$ of degree $n$, then

$$
\begin{aligned}
g(x) & =\sum_{k=0}^{n} \frac{1}{\alpha^{k}} \frac{1}{k !}\left(\Delta_{x}^{-, \alpha}\right)^{k} g(-k z) B_{k}(x, z) \\
& =\sum_{k=0}^{n} \frac{1}{\alpha^{k}} \frac{1}{k !}\left(\Delta_{x}^{-, \alpha}\right)^{k} g(-k z) x(x+\alpha+k z)^{\overline{k-1}, \alpha},
\end{aligned}
$$

where $\Delta_{x}^{-, \alpha}$ is a generalized backward difference with respect to $x$, given by $\Delta_{x}^{-, \alpha} g(x) \equiv g(x)-g(x-\alpha)$.

Proof of Theorem 1. We prove the equality (3). Let $f(x)$ be a polynomial in $x$ of degree $n f(x)=(x+y)^{\underline{n}, \alpha} \in \mathrm{C}[x]$. Then we get $\Delta_{x}^{+, \alpha} f(x)=n \alpha(x+y) \underline{n-1}, \alpha$ and $\left(\Delta_{x}^{+, \alpha}\right)^{2} f(x)=n(n-1) \alpha^{2}(x+y) \underline{n-2, \alpha}$. More generally we have

$$
\left(\Delta_{x}^{+, \alpha}\right)^{k} f(x)=n(n-1) \cdots(n-k+1) \alpha^{k}(x+y) \underline{n-k, \alpha}, \quad(k=1,2, \cdots, n) .
$$


From the equality (5) in Lemma 1, we have

$$
\begin{aligned}
(x+y)^{\underline{n}, \alpha} & =\sum_{k=0}^{n} \frac{1}{\alpha^{k}} \frac{1}{k !}\left(\Delta_{x}^{+, \alpha}\right)^{k} f(-k z) x(x-\alpha+k z) \underline{k-1}, \alpha \\
& =\sum_{k=0}^{n} \frac{1}{\alpha^{k}} \frac{1}{k !} n(n-1) \cdots(n-k+1) \alpha^{k}(-k z+y) \frac{n-k, \alpha}{x} x(x-\alpha+k z) \frac{k-1, \alpha}{} \\
& =\sum_{k=0}^{n}\left(\begin{array}{l}
n \\
k
\end{array}\right) x(x-\alpha+k z) \frac{k-1, \alpha}{2}(y-k z) \underline{n-k, \alpha}
\end{aligned}
$$

Therefore the equality (3) has been proved. The equality (4) can be also proven by the similar way using Lemma 2 .

\section{Acknowledgments}

The author (S. F.) was partially supported by JSPS KAKENHI Grant Number 24540146 .

\section{References}

[1] N.H. Abel, Beweis eines Ausdrucks von welchem die Binomial-Formel ein einzelner Fall ist, J. Reine Angew. Math., 1 (1826), 159-160.

[2] M. Itoh, Schur type functions associated with polynomial sequences of binomial type, Selecta Mathematica, 14 (2009), 247-274.

[3] K. Kuriyama, On an Abel's type generalization of the binomial theorems for the falling(or rising) factorial power, Mem. Fac. Edu. Bukkyo Univ., 13 (2014), 31-40.

[4] A. Nishi, S. Takigawa, On a generalization of binomial theorem by Abel (in Japanese), Mem. Fac. Edu. Saga Univ., 18 (2002), 39-43.

[5] G.C. Rota, D. Kahaner, A. Odlyzko, On the Foundations of Combinatorial Theory. VIII: Finite Operator Calculus, J. Math. Anal. Appl., 42 (1973), 684-760. 